\title{
Preparation of 2-Dimensional Ultra Thin Polystyrene Film by Water Casting Method
}

\author{
Kenshiro Shuto, Yushi Oishi, Tisato KaJiYama, ${ }^{\dagger}$ \\ and Charles C. HAN* \\ Department of Chemical Science and Technology, \\ Faculty of Engineering, Kyushu University, \\ 6-10-1 Hakozaki, Higashi-ku, Fukuoka 812, Japan \\ * Polymer Blends \& Solutions Group, Polymers Division, \\ National Institute of Standards \& Technology, \\ Gaithersburg, MD 20899, USA
}

(Received September 7, 1992)

\begin{abstract}
The preparation condition of polystyrene (PS) ultra thin film by a water casting method was investigated on the basis of transmission electron microscopic (TEM) observations. Homogeneous ultra thin films were prepared from a $0.2-2.0 \mathrm{wt} \%$ cyclohexanone solution of PS owing to moderate spreadability of the solution on the water surface. The spreading state of the solution on the water surface depending on the dissipation speed of solvent and entanglements among PS chains were in strong relation to formation of the homogeneous ultra thin film. X-Ray interference measurements revealed that the thickness of the ultra thin film was smaller than the dimension of an unperturbed PS chain. Such thinner film than the dimension of the unperturbed chain was defined as 2-dimensional ultra thin film in order to distinguish from 3-dimensional solid film. The radius of gyration of a polymer chain in the 2-dimensional ultra thin film was evaluated from small angle X-ray scattering (SAXS) and small angle neutron scattering (SANS) measurements. PS chains in the 2-dimensional ultra thin film expanded along the direction parallel to the film surface.

KEY WORDS Ultra Thin Polymer Film / Water Casting Method /

Polystyrene / Transmission Electron Microscopy / Small Angle X-Ray

Scattering / Tagged-Polymer Method / Small Angle Neutron Scattering /

Radius of Gyration /
\end{abstract}

Recently, ultra thin polymer films with thickness in the order of 10 to $100 \mathrm{~nm}$ have been developed into electrical and optical device for integrated circuits such as film insulator, nonlinear optical devices ${ }^{1}$ and optical waveguide, ${ }^{2}$ etc. In the development of the ultra thin polymer films, it is important to understand the aggregation state of polymer chains in the films. A flexible polymer chain behaves like an unperturbed chain in the 3-dimensional amorphous solid state. ${ }^{3}$ Thus, it is very important and interesting to study the case when the film thickness is smaller than the dimension of an unperturbed chain. This is because that the polymer chains in such system are restricted in a small spatial dimension perpendicular to the film surface so that the conformational entropy of an individual chain is smaller than that in a 3-dimensional solid state. It is expected that the aggregation structure and mechanical properties of films thinner than the dimension of unperturbed chain are different from those of the 3dimensional amorphous solid film. Therefore,

† To whom correspondence should be addressed. 
we should distinguish these particular cases from a 3-dimensional solid film. We define a polymer film thinner than the dimension of an unperturbed chain as a 2-demensional ultra thin film in order to distinguish it from a 3-dimensional solid film.

Ultra thin polymer film can be prepared by Langmuir-Blodgett, plasma polymerization, spin-coating and water casting methods. Among these methods, water casting method is especially useful because a ultra thin film with large area can be easily obtained even for a polymer which does not have any specific interaction with the water surface. Also the chemical structure of the polymer does not change during the process. Water casting film was widely used in cases such as surface alignment of films for liquid crystal orientation $^{4}$ and liquid crystal/polymer composite film for selective permeation of gas and ion. ${ }^{5}$ A successful preparation of water casting film depends on solvent, interfacial interaction between solution and water, surfactants used, and the temperature of the water surface, etc. ${ }^{4,5}$ And also, the preparation of water casting film may depend on concentration of solution, molecular weight of polymer and viscosity of solution, etc. However, formation mechanism of homogeneous ultra thin film by water casting method has not been clarified in detail.

In order to understand the formation mechanism of homogeneous 2-dimensional ultra thin film by water casting method, films prepared under various conditions were investigated by TEM observations and X-ray interference measurements. Then, the conformation of PS chains in these 2-dimensional ultra thin films were studied by X-ray and neutron scattering measurements.

\section{EXPERIMENTAL}

\section{Sample}

The samples used in the preparation of 2-dimensional ultra thin films in this study are all commercially available monodisperse polystyrenes with weight-average molecular weight, $M_{w}$, of 190000 (Tosoh Co., Ltd.), ${ }^{* 1} 233000$ (Pressure Chem. Co., Ltd.), 520000, 1030000, 1450000, and 6390000 (Polymer Lab. Co.) and $M_{w} / M_{n}$, where $M_{n}$ is number-average molecular weight, of 1.04, 1.06, 1.05, 1.05, 1.06, and 1.12 , respectively. For SANS measurements, deuterated PS (D-PS) with $M_{w}$ of 185000, 530000, and 1000000 (Polymer Lab. Co.), and $M_{w} / M_{n}$ of $1.02,1.09$, and 1.18 , respectively, were used.

\section{Preparation of 2-Dimensional Ultra Thin Film}

Figure 1 shows a schematic representation of the water casting method. The only procedure is a careful spreading of a single drop of polymer solution on the water surface. The
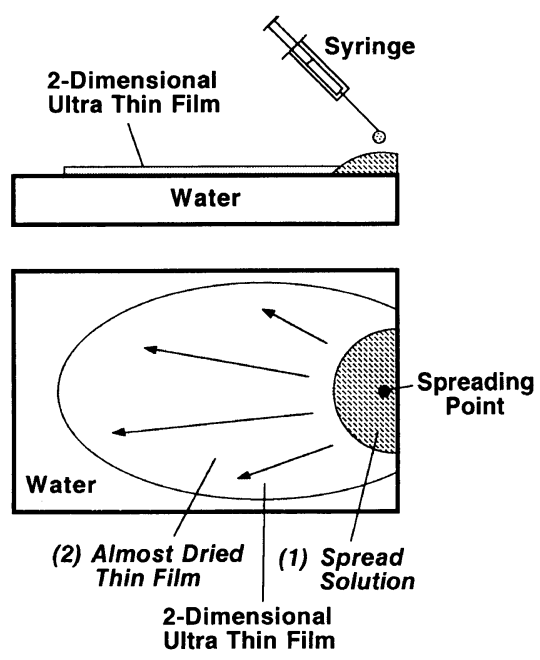

Figure 1. Schematic representation of water casting method. Initially (1) solution spreads and then (2) almost dried ultra thin polymer film comes out.

*1 Certain commercial equipment, instruments, or materials are identified in this paper in order to adequately specify the experimental procedure. Such identification does not imply recommendation or endorsement by the National Institute of Standards and Technology, nor does it imply that the materials or equipment identified are necessarily the best available for the purpose. 
polymer solution spreads automatically and then an almost dried thin film forms from the spread solution with dissipation of solvents by dissolution and evaporation. Spreading solutions were prepared from various good solvents for PS at concentrations of $0.1-2.0$ $w t \%$. These solutions were spread on the water surface at $293 \mathrm{~K}$. The ultra thin film was transferred onto an electron microscope grid coated with a collodion film. Pt-carbon was vapor-deposited onto the samples with a shadowing angle of 10 degree for the bright field electron microscopic observation. The intrinsic viscosity of solutions was measured using an Ubbelohde viscometer at $293 \mathrm{~K}$ to evaluate the cross-over concentration, $C^{*}$ between dilute and semi-dilute regions.

For the measurement of the thickness of the ultra thin film, $\mathrm{Cu}-K_{\alpha} \mathrm{X}$-ray beam was radiated to the films which were deposited on cover-glass substrate. The film transfer was accomplished by lowering the substrate to attach the film and then slowly withdrawing the substrate up after removing the surrounding film. Since the critical angles of the total reflection of X-ray at air-film and film-substrate interfaces in this case are about 0.2 and 0.5 degree, respectively, the interference peaks are strongly observed in a scattering angle region of $0.2-0.5$ degree. In order to evaluate the film thickness from the interference analysis, some interference peaks should be observed in this angle region, which requires the total thickness of around $200 \mathrm{~nm}$. Therefore, 6-10 films were deposited to form the layered film with the total thickness of around $200 \mathrm{~nm}$. Intensity of the X-ray interference was measured by a Rigaku SAXS diffractometer (Rigaku, CN2203E3) with a Kratky U-slit. A stabilized generator (Rigaku, Rota-Flex RU-200) operating at $50 \mathrm{kV}$ and $200 \mathrm{~mA}$ used as X-ray source.

\section{Evaluation of the Radius of Gyration of a Chain in 2-Dimensional Ultra Thin Film}

The radius of gyration of a polymer chain in the 2-dimensional ultra thin film was evaluated by SAXS measurements on the basis of a tagged-polymer method. ${ }^{6}$ The conformation of a tagged-PS in the matrix of regular PS chains can be studied from the analysis of the excess scattering due to the tagged-atom with high scattering power. A random copolymer chain composed of styrene and $p$-iodostyrene which was prepared by partial iodination of PS $^{7}$ with $M_{w}$ of 1450000 , was used as tagged-PS chain. The number fraction of $p$-iodostyrene monomer in the copolymer was $15.7 \%$. The sample for SAXS measurements was prepared by stacking 1000 ultra thin films together. As the sample of the 3-dimensional solid, thick film was prepared by solvent cast from a cyclohexanone solution. Samples consisting of regular PS together with $1.5 \mathrm{~mol} \%$ of taggedPS were prepared. The scattered intensity was measured with a SAXS diffractometer (Rigaku, CN2203E3) with a Kratky U-slit. A stabilized generator (Rigaku, Rota-Flex RU-200) operating at $50 \mathrm{kV}$ and $200 \mathrm{~mA}$ was used as X-ray source. The error from slit length was corrected by Schmidt's method. ${ }^{8}$ The excess scattering was plotted according to Guinier's procedure. ${ }^{9}$

$$
\begin{aligned}
& I(q)=I(0) \cdot \exp \left(-\frac{1}{3} q^{2} \cdot\left\langle S^{2}\right\rangle\right) \\
& q=\frac{4 \pi \sin \theta}{\lambda}
\end{aligned}
$$

where $q$ is the scattering vector, $I(q)$ and $I(0)$ are the excess scattered intensities at $q$ and $q=0$, respectively. From the initial slope of the plot of $\ln l(q) v s . q^{2}$, the radius of gyration, $\left\langle S^{2}\right\rangle^{1 / 2}$ of the tagged-PS was evaluated.

In order to evaluate the radius of gyration of a polymer chain in the 2-dimensional ultra thin film by SANS measurements, mixture of $20 \mathrm{vol} \%$ of D-PS and $80 \mathrm{vol} \%$ of PS were used as samples. Since the Flory's $\chi$ parameter between D-PS and PS is almost zero, ${ }^{10}$ the radius of gyration of a PS chain can be evaluated by fitting the Debye function to the excess scattering of the mixture after subtraction of the incoherent background from PS. 
Debye function is shown by eq 2 .

$$
\begin{aligned}
& S_{\text {Debye }}(u)=\frac{2}{u^{2}}\left(e^{-u}+u-1\right) \\
& u=q^{2} \cdot\left\langle S^{2}\right\rangle
\end{aligned}
$$

SANS intensity was measured by the $8 \mathrm{~m}$-SANS instrument at National Institute of Standards \& Technology (Gaithersburg, MD, USA) under the condition of wave length, $\lambda$ of $1.2 \mathrm{~nm}$ and camera length of $3.6 \mathrm{~m}$.

\section{RESULTS AND DISCUSSION}

\section{Preparation of 2-Dimensional Ultra Thin Film}

Figure 2 shows the electron micrographs of ultra thin films which have been prepared from $1 \mathrm{wt} \% n$-propylbenzene, cyclohexanone and methyl ethyl ketone solutions of PS with $M_{w}$ of 1450000. The bright field image of film prepared from cyclohexanone solution exhibited a uniform and homogeneous morphology, whereas those prepared from $n$-propylbenzene and methyl ethyl ketone solutions showed a heterogeneous morphology. In general, water casting films were formed through the processes of 1) spreading of solution and 2) appearance of a almost dried thin film, as shown in Figure 1. The cyclohexanone solution spread on the water surface and remain stable for a few second. On the contrary, the $n$-propylbenzene solution can not spread, which has resulted in the formation

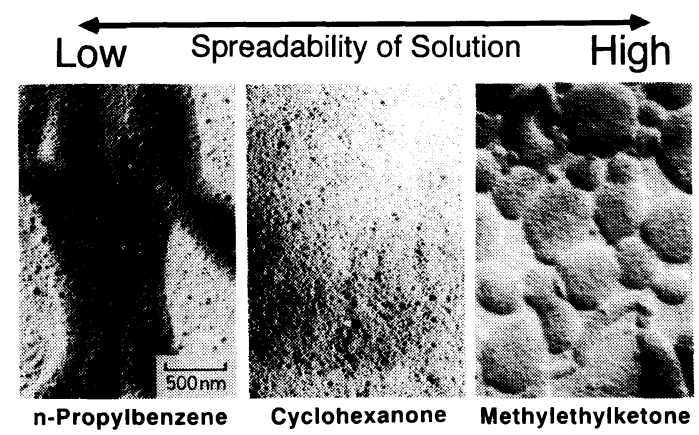

Figure 2. Transmission electron micrographs of ultra thin PS films prepared from $n$-propylbenzene, cyclohexanone, and methyl ethyl ketone solutions. of many small droplets, and the methyl ethyl ketone solution spreads rapidly and disappears immediately on the water surface. Then, it is apparent from Figure 2 that the moderate spreadability of a solution of the water surface is the key in the formation on a homogeneous film, whereas the excess or low spreadability of a solution forms a heterogeneous film. Therefore, it is clear that the type of water casting film which can be formed strongly depends on the phenomenal spreadability of the solution.

Figure 3 shows the classification of various spreading solvents on the basis of homogeneous or heterogeneous films on the water surface for a $1 \mathrm{wt} \%$ solution of PS with $M_{w}$ of 1450000. Open and filled circles represent spreading solvents forming homogeneous and heterogeneous films, respectively. The solutions prepared from solvents in the group 1 such as $n$-propylbenzene have fairly low spreadability on the water surface, solvents in the group 2 such as cyclohexanone have moderate spreadability and solvents in the group 3 such as methylethylketone have excess spreadability. Since the formation of homogenous film strongly depends upon the phenomenal spreadability of solution as shown in Figure 2, we have classified the solvent on the basis of interfacial interaction in equilibrium state without dissolution and evapora-

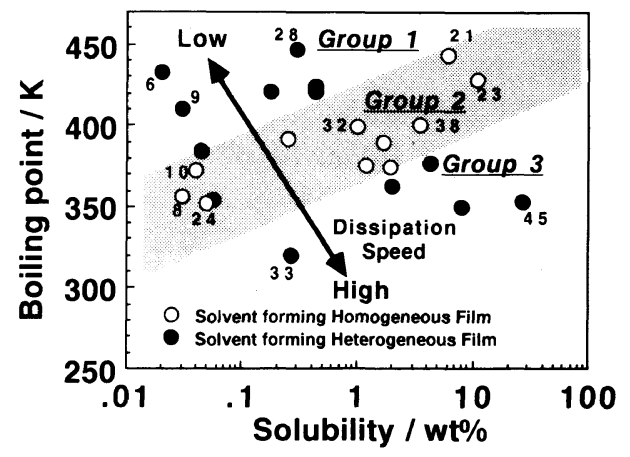

Figure 3. Classification of solvents. Open and filled circles represents solvents which formed homogeneous and heterogeneous film, respectively. Numbers for circles correspond to the magnitudes of $S_{1 / \mathrm{w}}$ in unit of $\mathrm{mN} \mathrm{m}^{-1}$. 
tion. $S_{1 / \mathrm{w}}$ is a spreading coefficient ${ }^{11}$ of a solvent which is determined from eq 3 .

$$
S_{\mathrm{l} / \mathrm{w}}=\gamma_{\mathrm{w}}-\left(\gamma_{1}+\gamma_{1 \mathrm{w}}\right)
$$

$\gamma_{w}, \gamma_{1}$, and $\gamma_{1 \mathrm{w}}$ are the surface tension of water, the surface tension of solvent, and the interfacial tension between solvent and water in equilibrium state, respectively. It has been generally known that solvents with large spreading coefficient expand well on the water surface. Therefore, the spreading coefficients of solvent should correspond to the spreadability of solution in this case, because the concentrations of all solutions used were the same and in the dilute region. However, as shown by the magnitudes of $S_{1 / \mathrm{w}}$ in Figure 3, there is no correlation between the $S_{1 / \mathrm{w}}$ of solvent and phenomenal spreadability of solutions. This seems to be caused by the rapid dissipation of solvent during the water casting process.

Next, we classified solvents on the basis of the dissipation speed from the water surface. The abscissa of Figure 3 is the solubility of spreading solvent in the water, which is assumed to be corresponding to the dissipation speed of spreading solvent by dissolution into water. The ordinate of Figure 3 is the boiling point of the spreading solvent, which may correspond to the magnitude of dissipation speed of solvent by evaporation into air. Thus, the dissipation speed of the spreading solvent into both water and air becomes lower or higher along the direction of arrows in the graph, and these arrows also correspond to the magnitude of spreadability of solutions. Therefore, it seems to be reasonable to conclude that the formation of homogeneous films is strongly related to the dissipation speed of spreading solvents into both air and water.

Figure 4 shows the electron micrographs of ultra thin films prepared from $0.1,0.2,0.5,1$, and $2 \mathrm{wt} \%$ cyclohexanone solutions of PS with $M_{w}$ of 1450000 . As mentioned above, a homogeneous water casting film can be formed by a cyclohexanone solution with moderate concentration. In this case homogeneous ultra

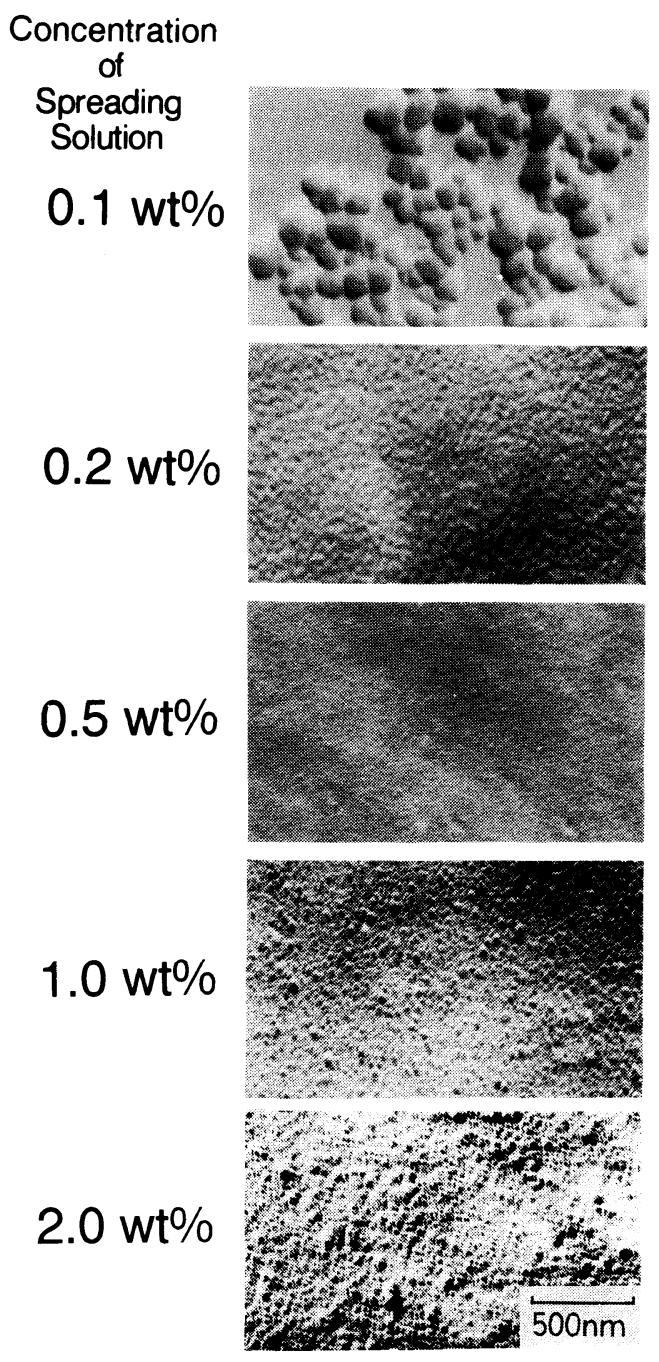

Figure 4. Transmission electron micrographs of ultra thin PS films prepared from $0.1-2.0 \mathrm{wt} \%$ cyclohexanone solutions.

thin films could be formed with solutions having concentration $0.2 \mathrm{wt} \%$ or above. On the other hand, particle aggregation was formed from $0.1 \mathrm{wt} \%$ solution. In other words, in the case of the cyclohexanone solution of PS with $M_{w}$ of 1450000 , the critical concentration above which homogeneous ultra thin film can be formed is about $0.1 \mathrm{wt} \%$. The critical concentrations for cyclohexanone and iso-pentylchloride solutions of PS with $M_{w}$ of 233000 , 
1450000 , and 6390000 are shown in Table I. The iso-pentylchloride solution formed homogeneous film on the water surface in a similar fashion to the cyclohexanone solution. It is speculated that polymer chains have to be entangled in order to form the ultra thin film during water casting process. In other words, when the polymer chains are not entangled during the spreading process to provide the integrity of the thin film, particle aggregation is formed. In order to confirm this speculation, the experimental concentrations in Table I were compared with cross-over concentration, $C^{*}$ as defined by eq 4 .

$$
C^{*}=\frac{M}{\frac{4}{3} \pi\left\langle S^{2}\right\rangle^{3 / 2} N_{\mathrm{A}}}
$$

where $\left\langle S^{2}\right\rangle, N_{\mathrm{A}}$ and $M$ are the mean square of the radius of gyration, the Avogadro's number, and the molecular weight, respectively. The intrinsic viscosity, $[\eta]$, is related to the radius of gyration by eq $5 .^{3}$

$$
\begin{aligned}
& {[\eta]=\phi \frac{\left\langle S^{2}\right\rangle^{3 / 2} \cdot 6^{3 / 2}}{M}} \\
& \phi=\phi^{0} \cdot \frac{\alpha_{\mathrm{s}}^{3}}{\alpha_{\eta}^{3}} \\
& \phi^{0}=2.5 \times 10^{21} \\
& \frac{\alpha_{\mathrm{s}}^{3}}{\alpha_{\eta}^{3}}=0.9
\end{aligned}
$$

$\phi$ and $\phi^{0}$ are the Flory-Fox viscosity constant for good solvent and that for $\theta$ solvent, respectively. Also, $\alpha_{\mathrm{s}}$ and $\alpha_{\eta}$ are the expansion ratio of the radius of gyration of a polymer chain in good solvent to that in $\theta$ solvent and the expansion ratio of the hydrodynamical radius of a polymer chain in good solvent to that in $\theta$ solvent, respectively. The values of $\phi^{0}$ and $\alpha_{s}^{3} / \alpha_{\eta}^{3}$ were referred from the detail experimental results about a PS solution reported by Yamakawa et al. ${ }^{12}$ From eq 4 and 5 , the $C^{*}$ is related to the intrinsic viscosity as given by eq 6 , under the assumption that the
Table I. Critical concentrations above which homogeneous film can be prepared

\begin{tabular}{ccc}
\hline \multirow{2}{*}{$M_{w}$} & Solvent & Critical concentration \\
\cline { 3 - 3 } & & $\mathrm{wt} \%$ \\
\hline \multirow{2}{*}{233000} & Cyclohexanone & 0.4 \\
& Isopentylchloride & 0.6 \\
\cline { 2 - 3 } 1450000 & Cyclohexanone & 0.1 \\
& Isopentylchloride & 0.3 \\
\cline { 2 - 3 } 6390000 & Cyclohexanone & 0.05 \\
\hline
\end{tabular}

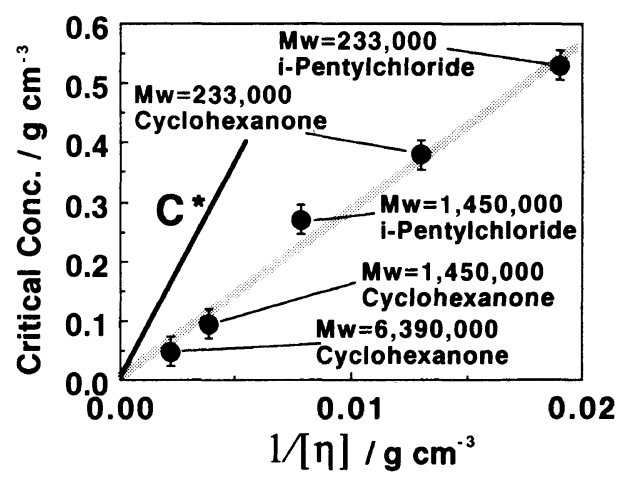

Figure 5. Relation between $[\eta], C^{*}$ and upper critical concentration above which homogeneous film can be prepared. Solid and broken lines show $C^{*}$ and critical concentration.

radius of gyration at $C^{*}$ is equal to that in dilute region.

$$
C^{*} \propto \frac{1}{[\eta]}
$$

Figure 5 shows the plot of the experimental critical concentrations and the calculated $C^{*}$ against the reciprocal of intrinsic viscosity. Broken and solid line represent the experimental critical concentration and the calculated $C^{*}$ from eq 6, respectively. As shown in Figure 5 , the experimental critical concentrations were also proportional to the reciprocal of intrinsic viscosity as the $C^{*}$ which is normally assumed as the onset of semidilute region above which entanglements among chains occur in the solution. The $1 /[\eta]$ dependence of the ex- 


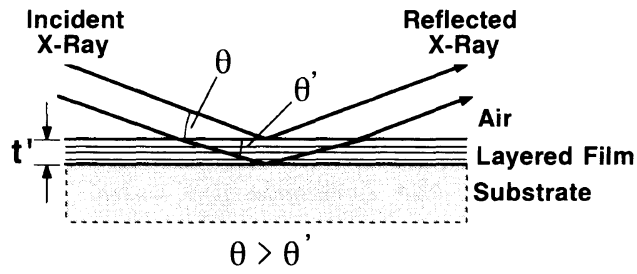

Figure 6. Schematic representation of X-ray interference method for evaluation of thickness. Reflected X-ray at air-film and film-substrate interfaces interfered each other.

perimental critical concentration for water casting of films are the same as $C^{*}$ however with smaller proportionality constant by a factor of 2, indicating that the critical concentration to form uniform film depends strongly on the entanglement of polymer chains. The factor of 2 difference in the $1 /[\eta]$ dependence of our critical concentration compare to $C^{*}$ may be an indication that the requirement of the degree of entanglement in film forming does not need to be very high.

Figure 6 illustrates the evaluation method of the total thickness of layered films. The interference peaks were observed by X-ray beams which were reflected from the air-film and the film-substrate interfaces. The interference peak angle, $\theta$ is related to the total thickness, $t^{\prime}$ of the layered film as given by eq 7.13

$$
\begin{aligned}
& 2 t^{\prime} \sin \theta\left(1-\frac{\delta}{\sin ^{2} \theta}\right)=n \cdot \lambda \\
& \delta=2.70 \times 10^{10} \cdot \lambda^{2} \cdot \rho \cdot \frac{\sum Z_{i}}{\sum A_{i}}
\end{aligned}
$$

$\lambda, 1-\delta$ and $\rho$ are the wavelength of X-ray, the refractive index of X-ray in the film, and the density of the film, respectively. Also, $Z_{i}$ and $A_{i}$ are the atomic mass and the atomic number, respectively.

Figure 7 shows a representative of the interference peaks marked with arrows for a 6-layered film prepared from a $2 \mathrm{wt} \%$ cyclohexanone solution of PS with $M_{w}$ of 1450000 . The interference peaks extent to many orders

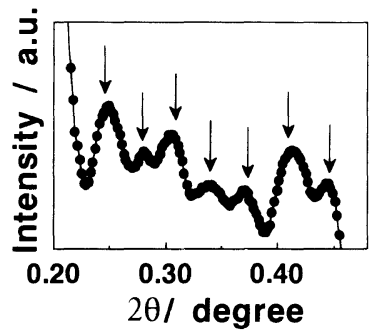

Figure 7. X-Ray interference intensity profiles from 6 films prepared from $2 \mathrm{wt} \%$ cyclohexanone solution. Arrows show interference peaks.

Table II. Observed and calculated interference peak angles of reflected X-ray from 6 films prepared from $2 \mathrm{wt} \%$ cyclohexanone solution

\begin{tabular}{ccc}
\hline & \multicolumn{2}{c}{ Peak angle $2 \theta /$ degree } \\
\cline { 2 - 3 } Peak order & Observed & Calculated \\
\hline 1 & 0.248 & 0.241 \\
2 & 0.282 & 0.271 \\
3 & 0.306 & 0.303 \\
4 & 0.340 & 0.339 \\
5 & 0.374 & 0.376 \\
6 & 0.410 & 0.415 \\
7 & 0.452 & 0.456 \\
\hline
\end{tabular}

indicate that the thickness distribution in the film was narrow, in other words, the film surface was flat over a large area. Table II shows the observed peak angles and the calculated peak angles on the assumption that the total thickness of the 6-layered film is $175 \mathrm{~nm}$. The observed peak angles agreed quite well with the calculated peak angles, indicating that the thickness of the ultra thin film prepared from $2 \mathrm{wt} \%$ cyclohexanone solution was about $29 \mathrm{~nm}$

Table III shows the thicknesses evaluated by the above method for thin films prepared from $0.3-2.0 \mathrm{wt} \%$ solutions. It is reported that $\left\langle R_{0}^{2}\right\rangle \mid N \cdot b^{2}$ of PS is 10.6 , in which $\left\langle R_{0}^{2}\right\rangle, N$ and $b$ are the mean square end-to-end distance of an unperturbed chain, the number of segments in a chain and the segment length per one $\mathrm{C}-\mathrm{C}$ bond in a main chain, respectively. ${ }^{14}$ The end-to-end distance of an unperturbed PS 
chain with $M_{w}$ of 1450000 is about $80 \mathrm{~nm}$. The end-to-end distance is regarded as a representation of the dimension of a chain, then the thickness of ultra thin films listed in Table III is smaller than the dimension of unperturbed PS chain mentioned above. Thus, a 2-dimensional ultra thin film as defined previously can be easily prepared by this water casting method. As given in Table III, the thickness of the film decreases with the concentration of spreading solution. Therefore, it should be noted that the thickness of a 2-dimensional ultra thin film can be controlled by the concentration of the spreading solution.

\section{Conformation of a Polymer Chain in 2-} Dimensional Ultra Thin Film

Table IV shows the radius of gyration of a polymer chain in the 2-dimensional ultra thin film and the 3-dimensional solid film measured

Table III. Thickness of 2-dimensional ultra thin film prepared from cyclohexanone solution at various concentration

\begin{tabular}{cc}
\hline $\begin{array}{c}\text { Concentration } \\
\text { of solution }\end{array}$ & $\begin{array}{c}\text { Thickness } \\
\text { of the film }\end{array}$ \\
\cline { 2 - 2 } wt $\%$ & $\mathrm{~nm}$ \\
\hline 0.3 & 11 \\
0.5 & 14 \\
1.0 & 19 \\
2.0 & 29 \\
\hline
\end{tabular}

by the SANS and SAXS experiments. $\left\langle R^{2}\right\rangle_{3 \mathrm{D}}^{1 / 2}$, $\left\langle S^{2}\right\rangle_{3 \mathrm{D}}^{1 / 2}$, and $\left\langle S^{2}\right\rangle_{2 \mathrm{D}}^{1 / 2}$ are the end-to-end distance of a polymer chain in the 3dimensional solid film which is calculated as $6^{1 / 2} \cdot\left\langle S^{2}\right\rangle_{3 \mathrm{D}}^{1 / 2}$, the experimental value of the radius of gyration in the 3-dimensional solid film, and that of the radius of gyration in the 2-dimensional ultra thin film, respectively. Since X-ray and neutron beams were irradiated perpendicular to the film surface, the evaluated values of the radius of gyration, $\left\langle S^{2}\right\rangle_{2 D}^{1 / 2}$ corresponds to those along the direction parallel to the film surface. As shown in Table IV, the value of $\left\langle S^{2}\right\rangle_{2 \mathrm{D}}^{1 / 2}$ is larger than that of $\left\langle S^{2}\right\rangle_{3 \mathrm{D}}^{1 / 2}$. This indicates that a polymer chain in the 2-dimensional ultra thin film prepared by the water casting method is expanded along the direction parallel to the film surface. Strictly speaking, the values of $\left\langle S^{2}\right\rangle_{3 \mathrm{D}}^{1 / 2}$ and $\left\langle S^{2}\right\rangle_{2 \mathrm{D}}^{1 / 2}$ which were evaluated by SAXS are not the radius of gyration of a PS chain but that of a tagged-PS chain. According to Hamada et al. ${ }^{6}$ the exact value of radius of gyration of a PS chain must be determined from an extrapolation to both zero iodine contents and the zero fraction of tagged-PS. However, it is reasonable to consider that the radius of gyration in the 2-dimensional ultra thin film can be compared relative to that in a 3 -dimensional solid film, because the conformation of a tagged-PS chains is close to that of a PS chain. ${ }^{6}$

Figure 8 shows the schematic representation

Table IV. Radius of gyration of a polystyrene chain along the direction parallel to film surface evaluated by SANS and SAXS

\begin{tabular}{|c|c|c|c|c|c|}
\hline \multirow{2}{*}{ Method } & \multirow{2}{*}{$M_{w}$} & $t$ & $\left\langle R^{2}\right\rangle_{3 \mathrm{D}}^{1 / 2}$ & $\left\langle S^{2}\right\rangle_{3 \mathrm{D}}^{1 / 2}$ & $\left\langle S^{2}\right\rangle_{2 \mathrm{D}}^{1 / 2}$ \\
\hline & & $\mathrm{nm}$ & $\mathrm{nm}$ & $\mathrm{nm}$ & $\mathrm{nm}$ \\
\hline \multirow{4}{*}{ SANS } & 1000000 & 19 & 67.4 & 27.5 & 40.2 \\
\hline & 530000 & 19 & 47.5 & 19.4 & 27.8 \\
\hline & 185000 & 19 & 30.2 & 12.3 & 15.0 \\
\hline & 185000 & 29 & 30.2 & 12.3 & 13.6 \\
\hline \multirow{3}{*}{ SAXS } & 1450000 & 14 & 84 & 34 & 52 \\
\hline & 1450000 & 19 & 84 & 34 & 48 \\
\hline & 1450000 & 29 & 84 & 34 & 42 \\
\hline
\end{tabular}




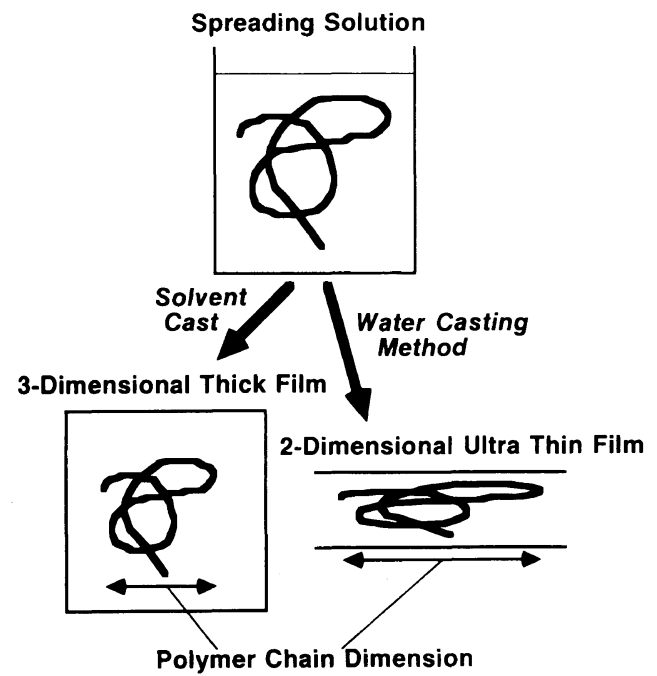

Figure 8. Conformation model of polymer chain in 2-dimensional ultra thin film prepared by water casting method.

of a polymer chain conformation in the 2-dimensional ultra thin film prepared by water casting method and in the 3-dimensional solid film prepared by solvent cast method. The dimension of a PS chain in the 2-dimensional ultra thin film along the direction parallel to the film surface is larger than that in the 3-dimensional solid film. The conformation of a polymer chain in the 2-dimensional state has been investigated on the basis of theoretical and numerical studies. ${ }^{15-19}$ In the case of an unperturbed, Gaussian, chain with no excluded volume, the segment density distribution along one direction (e.g., parallel to the film surface) is independent of that along the other direction (e.g., perpendicular to the film surface). This means that the segment density distribution of a chain in the direction parallel to the film surface dose not change, even if the segment density distribution along the direction perpendicular to the film surface is restricted to a small spatial dimension. Thus, the radius of gyration of an unperturbed chain along the direction parallel to the film surface should be equal to that of an unperturbed chain in the 3dimensional state. On the contrary, in the case of a perturbed chain with excluded volume, the excluded volume affecting the segment density distribution along the direction parallel to the film surface is augmented by that along the direction perpendicular to the film surface, when the perturbed chain is restricted in a small spatial dimension along the direction perpendicular to the film surface. Therefore, the perturbed chain dimension along the direction parallel to the film surface becomes larger than the perturbed chain dimension in 3-dimensional state. Since $\left\langle S^{2}\right\rangle_{2 \mathrm{D}}^{1 / 2}$ is larger than $\left\langle S^{2}\right\rangle_{3 \mathrm{D}}^{1 / 2}$ as shown in Table IV, it is reasonable to conclude that a PS chain in the 2dimensional ultra thin film prepared by water casting method dose not behave like an unperturbed chain in the 2-dimensional state. Some origins for the excess expansion of a PS chain along the direction parallel to the film surface are speculated as follows. First is the excluded volume effect which may be caused by the higher segment density of a chain in the 2-dimensional state than that of the 3dimensional state, due to the restricted dimension of the polymer chain which cause the decrease of apparent volume of a chain and the increase of the number of contact of segments of a chain. Second is freezing of expanded chains during the process of film formation by rapid biaxial of the solution and the dissipation of solvent. Further discussion of the conformation of a polymer chain in the 2-dimensional ultra thin film prepared by water casting method is necessary, and will be done elsewhere.

\section{CONCLUSION}

Morphological homogeneous ultra thin PS film was prepared by the water casting method. The thickness of the ultra thin film can be smaller than the end-to-end distance of the unperturbed PS chain and also, this thickness is controlled by the concentration of the spreading solution. A 2-dimensional ultra thin film is defined as a thin film which has a film 
thickness thinner than the dimension of the unperturbed polymer chain. In the process of water casting, first the solution is spreaded on the water surface depending on the dissipation speed of the solvent into both air and water, and then almost dried thin film can be obtained. During the formation process, the entanglement among chains keeps the integrity of the 2-dimensional ultra thin film. A PS chain in a 2-dimensional ultra thin film prepared by the water casting method is expanded along the direction parallel to the film surface.

\section{REFERENCES}

1. See for example, A. J. Heeger, D. Moses, and M. Sinclair, Synth. Met., 17, 343 (1987).

2. See for example, R. Ulrich and H. P. Weber, Appl. Opt., 11, 428 (1972).

3. P. J. Flory, J. Chem. Phys., 17, 303 (1949).

4. N. Masutani and I. Sakai, Sen-i Gakkaishi, 46(2), 47 (1990).
5. T. Kajiyama, H. Kikuchi, and S. Shinkai, J. Membrane Sci., 36, 243 (1988).

6. F. Hamada, H. Hayashi, and A. Nakajima, J. Appl. Cryst., 11, 514 (1979).

7. D. Braun, Makromol. Chem., 30, 85 (1959).

8. P. W. Schmidt and R. Hight, Jr., Acta Cryst., 13, 480 (1960).

9. A. Guinier, J. Chem. Phys., 40, 133 (1943).

10. H. Yang, R. S. Stein, C. C. Han, B. J. Bauer, and E. J. Kramer, Polym. Commun., 27, 132 (1986).

11. W. Adamson, "Physical Chemistry of Surfaces," 4th ed, J. Wiley \& Sons, Inc., New York, N.Y., 1982, p 100 .

12. A. Yamamoto, M. Fuji, G. Tanaka, and $\mathrm{H}$. Yamakawa, Polym. J., 2, 799 (1971).

13. L. G. Parratt, Phys. Rev., 95, 359 (1954).

14. W. R. Krigbaum, J. E. Kurz, and P. Smith, J. Phys. Chem., 65, 1984 (1961).

15. E. A. Dimarzio and R. J. Rubin, J. Chem. Phys., 55, 4318 (1971).

16. M. Daoud and P. G. De Gennes, J. Physique, 38, 85 (1977).

17. P. G. De Gennes, Macromolecules., 15, 492 (1982).

18. T. Ishinabe, J. Chem. Phys., 83, 423 (1985).

19. K. Shiokawa, Polym. J., 22, 925 (1990). 